K. M. Utzschneider • D. B. Carr · J. Tong •

T. M. Wallace $\cdot$ R. L. Hull $\cdot$ S. Zraika $\cdot$ Q. Xiao $\cdot$

J. S. Mistry • B. M. Retzlaff • R. H. Knopp · S. E. Kahn

\title{
Resistin is not associated with insulin sensitivity or the metabolic syndrome in humans
}

Received: 7 June 2005 / Accepted: 19 June 2005 / Published online: 6 September 2005

C) Springer-Verlag 2005

\begin{abstract}
Aims/hypothesis: The aim of this study was to further elucidate the relationship between resistin and insulin sensitivity, body fat distribution and the metabolic syndrome in humans. Methods: We measured plasma resistin levels in 177 non-diabetic subjects $(75$ male, 102 female; age 32-75 years). BMI, waist circumference, blood pressure, lipids, glucose, plasminogen-activator inhibitor 1 (PAI-1), adiponectin and leptin levels were also measured. The insulin sensitivity index $\left(S_{\mathrm{I}}\right)$ was quantified using Bergman's minimal model. Intra-abdominal fat (IAF) and subcutaneous fat (SQF) areas were quantified by CT scan. Presence of metabolic syndrome criteria was determined using the National Cholesterol Education Program Adult Treatment Panel III guidelines. Results: When subjects were divided into categories based on BMI $(<$ or $\left.\geq 27.5 \mathrm{~kg} / \mathrm{m}^{2}\right)$ and $S_{\mathrm{I}}\left(<\right.$ or $\left.\geq 7 \times 10^{-5} \mathrm{~min}^{-1}[\mathrm{pmol} / 1]^{-1}\right)$, resistin levels did not differ between the lean, insulinsensitive $(n=53,5.36 \pm 0.3 \mathrm{ng} / \mathrm{ml})$, lean, insulin-resistant $(n=67,5.70 \pm 0.4 \mathrm{ng} / \mathrm{ml})$ and obese, insulin-resistant
\end{abstract}

K. M. Utzschneider · J. Tong · T. M. Wallace $\cdot$ R. L. Hull · S. Zraika - B. M. Retzlaff - R. H. Knopp - S. E. Kahn Division of Metabolism, Endocrinology and Nutrition, Department of Medicine, Veterans Affairs Puget Sound Health Care System and Harborview Medical Center, University of Washington,

Seattle, WA, USA

D. B. Carr

Division of Maternal-Fetal Medicine,

Department of Obstetrics and Gynecology,

University of Washington,

Seattle, WA, USA

Q. Xiao · J. S. Mistry

Linco Research,

St Charles, MO, USA

K. M. Utzschneider $(\bowtie)$

Veterans Affairs Puget Sound Health Care System (151),

1660 S. Columbian Way,

Seattle, WA 98108, USA

e-mail: kutzschn@u.washington.edu

Tel.: +1-206-277-3568

Fax: +1-206-277-3011 groups $(n=48,5.94 \pm 0.4 \mathrm{ng} / \mathrm{ml}$; ANOVA $p=0.65)$. Resistin correlated with age $(r=-0.22, p<0.01)$, BMI $(r=0.16$, $p=0.03)$ and SQF $(r=0.19, p=0.01)$ but not with $S_{\mathrm{I}}$ $(p=0.31)$ or IAF $(p=0.52)$. Resistin did not correlate with the number of metabolic syndrome criteria or any of the individual metabolic syndrome criteria. In contrast, adiponectin, PAI-1 and leptin each correlated with IAF, SQF and $S_{\mathrm{I}}$. Additionally, the number of metabolic syndrome criteria correlated with adiponectin $(r=-0.32$, $p<0.001)$, leptin $(r=0.31, p<0.001)$ and PAI- $1(r=0.26$, $p=0.001)$. Conclusions/interpretation: In contrast to other adipokines, resistin is only weakly associated with body fat and is unlikely to be a major mediator of insulin resistance or the metabolic syndrome in humans.

Keywords Adiponectin - Insulin resistance · Insulin sensitivity · Leptin · Metabolic syndrome · Plasminogen-activator inhibitor $1 \cdot$ Resistin

Abbreviations IAF: intra-abdominal fat - LIS: lean, insulin-sensitive - PAI-1: plasminogen-activator inhibitor 1 . $\mathrm{S}_{\mathrm{I}}$ : insulin sensitivity index $\cdot \mathrm{SQF}$ : subcutaneous fat

\section{Introduction}

Resistin was discovered as an adipose-derived hormone in mice and has been postulated to play a role in the relationship between obesity and insulin resistance [1]. Although the data in mice are quite compelling, human data on the role of resistin in metabolism have been inconsistent. For example, the relationship between resistin levels and obesity has been inconsistent and little is known about the relationship between resistin and central body fat distribution [2]. Human studies have also failed to find significant correlations between resistin and measures of insulin sensitivity [3,4]. Whether resistin is associated with the metabolic syndrome is not clear. In contrast, other adipokines, such as leptin, adiponectin and plasminogen 
activator inhibitor 1 (PAI-1), have been found to be clearly associated with insulin sensitivity, body fat distribution and the metabolic syndrome in humans [5].

Given that a definitive conclusion regarding the relationship between resistin and human metabolism is yet to be reached, we undertook a study in a large cohort of apparently healthy subjects in order to determine whether resistin levels in humans are associated with obesity, central body fat distribution, insulin sensitivity and the metabolic syndrome.

\section{Subjects and methods}

Subjects Plasma resistin levels were measured in 177 subjects (75 male, 102 female; age 32-75 years) who participated in a study of the effects of insulin sensitivity on the plasma lipid profile response to egg feeding [6]. All subjects provided written informed consent to participate in the study, which was reviewed and approved by the Human Subjects Review Committee at the University of Washington.

Body composition and insulin sensitivity The following variables were measured as previously described [6]: intraabdominal fat (IAF) and abdominal subcutaneous fat (SQF) areas by CT scan at the level of the umbilicus; and the insulin sensitivity index $\left(S_{\mathrm{I}}\right)$ from a tolbutamide-modified frequently sampled IVGTT using Bergman's minimal model of glucose kinetics.

Assays Plasma resistin and PAI-1 levels were quantified using the Human Adipokine LINCOplex assay kit (Linco Research, St Charles, MO, USA). The antibody pairs used for each analyte in the multiplex panel are highly specific and no significant cross-reactivity within the panel was observed. The sensitivity of the assay is $6.7 \mathrm{pg} / \mathrm{ml}$ for resistin and $1.3 \mathrm{pg} / \mathrm{ml}$ for PAI-1. Intra-assay variation for resistin and PAI-1 levels was $2-8 \%$ and $2-5 \%$ and interassay variation for resistin and PAI-1 levels was $6-13 \%$ and $5-10 \%$. The average recovery for the linearity of dilution is $99.8 \%$ and the recovery of spiked adipokines to the samples containing endogenous levels of adipokines was $90-121 \%$ for resistin and $96-107 \%$ for PAI-1. Fasting plasma leptin and adiponectin concentrations were measured by RIA (Linco Research).

Resistin and PAI-1 concentrations were measured using the 3-min sample from the frequently sampled IVGTT due to lack of adequate volume from the fasting sample. In a pilot study, we determined that resistin and PAI-1 levels did not differ when measured before and $3 \mathrm{~min}$ after glucose administration (data not shown).

Metabolic syndrome criteria Data were analysed in 164 subjects in whom we had measured all five metabolic syndrome criteria according to the National Cholesterol Education Program Adult Treatment Panel III guidelines. These criteria are defined as blood pressure $\geq 130 / 85 \mathrm{mmHg}$, waist circumference $>102 \mathrm{~cm}$ in men and $>88 \mathrm{~cm}$ in women, HDL-cholesterol $<1.03 \mathrm{mmol} / \mathrm{l}$ in men and $<1.29 \mathrm{mmol} / \mathrm{l}$ in women, triglycerides $\geq 1.69 \mathrm{mmol} / \mathrm{l}$, and fasting plasma glucose $\geq 6.1 \mathrm{mmol} / 1$.

Statistical analysis Student's $t$-test or ANOVA with Bonferroni post-hoc analysis was performed to ascertain differences among groups. Simple or multiple linear regression analysis was performed to determine correlations between measures of interest. Data that were not normally distributed were transformed to achieve normal distribution. Data are presented as means \pm SEM unless otherwise specified. A two-sided $p<0.05$ was considered statistically significant.

\section{Results}

Subject characteristics by sex Subjects demonstrated a wide range of age (32-75 years), BMI $\left(18.4-46.7 \mathrm{~kg} / \mathrm{m}^{2}\right)$ and insulin sensitivity $\left(S_{\mathrm{I}}\right.$ : $\left.[0.7-30.0] \times 10^{-5} \mathrm{~min}^{-1}[\mathrm{pmol} / 1]^{-1}\right)$. Resistin levels were not significantly different between men and women, but PAI-1 levels were significantly higher in men, and leptin and adiponectin levels were higher in women (Fig. 1a).

Subject characteristics by BMI and insulin sensitivity Subjects were divided into four groups based on BMI and insulin sensitivity (BMI $<$ or $\geq 27.5 \mathrm{~kg} / \mathrm{m}^{2}$ and $S_{\mathrm{I}}<$ or $\geq 7.0 \times 10^{-5} \mathrm{~min}^{-1}[\mathrm{pmol} / 1]^{-1}$ ). Because only nine subjects were classified as obese, insulin-sensitive they were not included in the analysis. Exclusion of these subjects did not affect the results. Resistin levels did not differ between the lean, insulin-sensitive (LIS) group, the lean, insulin-resistant group and the obese, insulin-resistant group (Fig. 1b). In contrast, adiponectin levels were lower in both insulin-resistant groups compared with the LIS group and leptin and PAI-1 levels were higher in the obese group compared with the lean groups (Fig. 1b).

Relationships between adipokines, age, insulin sensitivity and body fat measures As listed in Table 1, resistin levels were strongly associated with age, weakly associated with BMI and SQF but not associated with $S_{\text {I }}$ or IAF. In contrast, adiponectin, leptin and PAI-1 levels demonstrated significant associations with $S_{\mathrm{I}}$ and IAF and were more strongly associated with BMI and SQF than resistin. Adiponectin, but not leptin or PAI-1, was related to age.

In a multiple regression model, adjusting for age and sex, BMI was independently correlated with resistin levels $(r=0.17, p<0.05)$, adiponectin $(r=0.32, p<0.001)$, age $(r=0.28, p<0.001)$, leptin $(r=0.69, p<0.001)$ and PAI-1 $(r=0.38, p<0.001)$.

With insulin sensitivity as the dependent variable and the individual adipokines, age, sex and BMI as the independent variables, adiponectin $(r=0.34, p<0.001)$, leptin $(r=-0.35, p<0.001)$ and PAI-1 $(r=-0.29, p<0.001)$ remained independently associated with $S_{\mathrm{I}}$, but resistin was not $(p=0.48)$. When IAF and SQF were substituted for BMI in the regression models the findings were similar. 
a

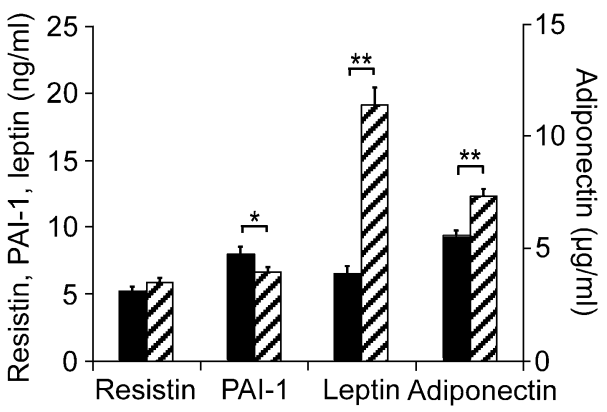

b

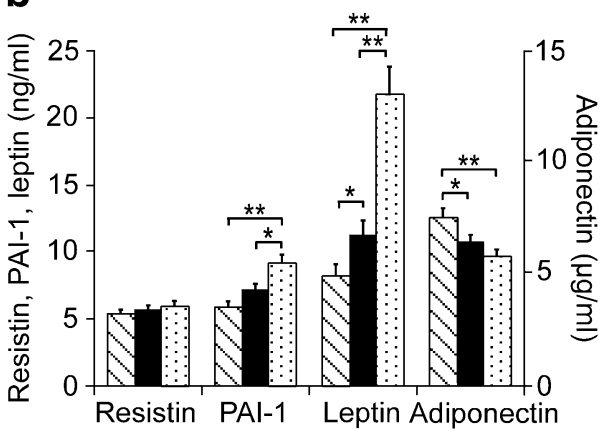

C

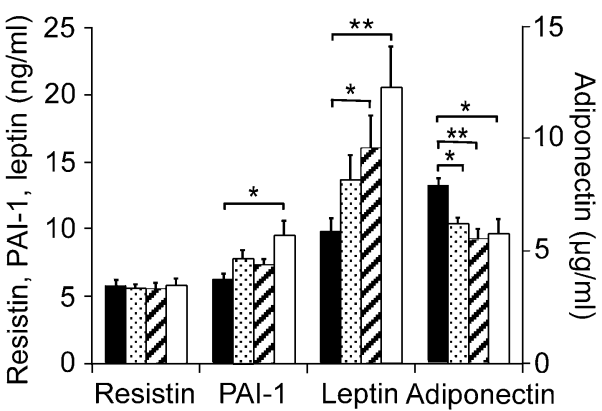

Fig. 1 Levels of adipokines by (a) sex (men, solid bar, $n=75$; women, striped bar, $n=102$ ), (b) BMI and $S_{\text {I }}$ (lean, insulin-sensitive, striped bar, $n=53$; lean, insulin-resistant, solid bar, $n=67$; obese, insulin-resistant, dotted bar, $n=48$ ), and c number of metabolic syndrome criteria $(0$, solid bar, $n=64 ; 1$, dotted bar, $n=47 ; 2$, striped bar, $n=33$; and $\geq 3$ criteria, open bar, $n=20)$. Data are presented as means \pm SEM. ${ }^{*} p<0.05, * * p<0.001$ between groups

Adipokine levels relative to metabolic syndrome criteria When analysed by number of metabolic syndrome criteria, resistin levels did not differ (Fig. 1c). In contrast, levels of leptin increased and adiponectin decreased in subjects with two or more criteria compared with those with none. PAI-1 was increased in subjects with three or more criteria (Fig. 1c). The number of metabolic syndrome criteria did not correlate with resistin levels $(r=0.006, p=0.9)$ but was significantly correlated with adiponectin $(r=-0.32, p<0.001)$, leptin $(r=0.31, p<0.001)$ and PAI-1 $(r=0.26, p=0.001)$. There were no significant correlations between resistin levels and any of the individual metabolic syndrome criteria.

Relationships between resistin and other adipokines Resistin did not correlate with adiponectin, but was positively correlated with leptin $(r=0.20, p<0.01)$. This association became non-significant when adjusted for age, sex and BMI. Resistin also correlated with PAI-1 $(r=0.31, P<0.001)$ and this relationship remained after adjustment for age, sex and BMI (partial $r=0.32, P<0.001$ ).

\section{Discussion}

We have demonstrated that resistin levels were not associated with IAF, insulin sensitivity or the metabolic syndrome and were only weakly associated with BMI and SQF. Our findings serve to clarify the previous literature regarding resistin's relationship with obesity and central body fat distribution, and are in agreement with previous findings of a lack of an association between resistin and insulin sensitivity in humans $[3,4]$. We have further extended these observations by assessing for the first time the relationship between resistin levels and the individual metabolic syndrome criteria. Collectively, our data suggest that resistin is unlikely to play an important role in the constellation of clinical findings frequently associated with insulin resistance in humans.

While we found a weak relationship between resistin and BMI, resistin was not associated with IAF, the fat depot most strongly associated with insulin sensitivity [6]. Only one previous study has investigated the relationship between resistin and abdominal fat distribution by CT scan [2]. In their Japanese cohort, Azuma et al. [2] found higher resistin levels in the overweight-to-obese subjects when compared with lean subjects, but no correlation between resistin levels and IAF or SQF. Our study adds to this literature by demonstrating no relationship between resistin levels and IAF in a larger, predominantly Caucasian cohort.

We also found that resistin levels did not differ with increasing number of metabolic syndrome criteria and were not correlated with any of the individual metabolic syn-
Table 1 Simple linear regression models evaluating the association between the adipokines and age, $S_{\mathrm{I}}$ and body fat measures.

\begin{tabular}{|c|c|c|c|c|c|c|c|c|}
\hline & \multicolumn{2}{|c|}{ Resistin } & \multicolumn{2}{|c|}{ Adiponectin } & \multicolumn{2}{|l|}{ Leptin } & \multicolumn{2}{|l|}{ PAI-1 } \\
\hline & $r$ & $p$ value & $r$ & $p$ value & $r$ & $p$ value & $r$ & $p$ value \\
\hline Age & -0.21 & 0.005 & 0.25 & 0.001 & 0.03 & 0.7 & -0.01 & 0.9 \\
\hline$S_{\text {I }}$ & 0.08 & 0.3 & 0.40 & $<0.001$ & -0.38 & $<0.001$ & -0.43 & $<0.001$ \\
\hline BMI & 0.15 & $<0.05$ & -0.34 & $<0.001$ & 0.49 & $<0.001$ & 0.39 & $<0.001$ \\
\hline SQF & 0.18 & $<0.05$ & -0.18 & $<0.05$ & 0.75 & $<0.001$ & 0.25 & 0.001 \\
\hline IAF & 0.05 & 0.5 & -0.35 & $<0.001$ & 0.28 & $<0.001$ & 0.43 & $<0.001$ \\
\hline
\end{tabular}


drome criteria. Our findings contrast with data from the Study of Inherited Risk of Coronary Atherosclerosis, in which subjects with the metabolic syndrome $(n=249)$ had marginally higher resistin levels than those without $(n=630$; 5.72 vs $5.41 \mathrm{ng} / \mathrm{ml}, p=0.03$ ) [7]. However, the clinical significance of such small differences in resistin levels, demonstrated only in a large cohort, is not clear.

One possibility for the lack of a strong association between resistin and obesity could be that in humans, macrophages rather than adipocytes are the major source of resistin [8]. The link between resistin and inflammation is supported by studies in humans demonstrating increased resistin levels with acute inflammation [9] and positive associations between resistin levels and inflammatory markers $[7,10]$. The association that we found between resistin and PAI-1 levels supports the hypothesis that resistin may play more of an inflammatory rather than a metabolic role in humans. It is also possible that resistin may have unidentified local effects which cannot be elucidated by measuring plasma levels.

Duality of interest The co-authors Q. Xiao and J. S. Mistry work for Linco Research. None of the other authors has a conflict of interest with this research to declare.

Acknowledgements We thank the subjects who participated in the study, and D. Collins and the nursing staff of the General Clinical Research Center at the University of Washington for their care of the subjects. This work was supported in part by the Medical Research Service of the Department of Veterans Affairs, the American Egg Board, NIH grants DK-02654, DK-17047, DK-35747, DK-35816, HL-30086, HL-07028, RR-37 and RR-16066, the American Diabetes Association, the US Department of Agriculture, and the McMillen Family Trust. S. E. Kahn is a recipient of a Distinguished Clinical Scientist Award from the American Diabetes Association.

\section{References}

1. Steppan CM, Bailey ST, Bhat S et al (2001) The hormone resistin links obesity to diabetes. Nature 409:307-312

2. Azuma K, Katsukawa F, Oguchi S et al (2003) Correlation between serum resistin level and adiposity in obese individuals. Obes Res 11:997-1001

3. Volarova de Courten B, Degawa-Yamauchi M, Considine RV, Tataranni PA (2004) High serum resistin is associated with an increase in adiposity but not a worsening of insulin resistance in Pima Indians. Diabetes 53:1279-1284

4. Heilbronn LK, Rood J, Janderova L et al (2004) Relationship between serum resistin concentrations and insulin resistance in nonobese, obese, and obese diabetic subjects. J Clin Endocrinol Metab 89:1844-1848

5. Kershaw EE, Flier JS (2004) Adipose tissue as an endocrine organ. J Clin Endocrinol Metab 89:2548-2556

6. Carr DB, Utzschneider KM, Hull RL et al (2004) Intraabdominal fat is a major determinant of the National Cholesterol Education Program Adult Treatment Panel III criteria for the metabolic syndrome. Diabetes 53:2087-2094

7. Reilly MP, Lehrke M, Wolfe ML, Rohatgi A, Lazar MA, Rader DJ (2005) Resistin is an inflammatory marker of atherosclerosis in humans. Circulation 111:932-939

8. Patel L, Buckels AC, Kinghorn IJ et al (2003) Resistin is expressed in human macrophages and directly regulated by PPAR gamma activators. Biochem Biophys Res Commun 300:472-476

9. Lehrke M, Reilly MP, Millington SC, Iqbal N, Rader DJ, Lazar MA (2004) An inflammatory cascade leading to hyperresistinemia in humans. Plos Med 1:e45

10. Shetty GK, Economides PA, Horton ES, Mantzoros CS, Veves A (2004) Circulating adiponectin and resistin levels in relation to metabolic factors, inflammatory markers, and vascular reactivity in diabetic patients and subjects at risk for diabetes. Diabetes Care 27:2450-245 